\section{(O) OPEN ACCESS}

PAPER

\title{
The potential benefit of the placebo effect in sham-controlled trials: implications for risk-benefit assessments and informed consent
}

\author{
Remy L Brim, Franklin G Miller
}

Department of Bioethics, National Institutes of Health, Bethesda, Maryland, USA

\section{Correspondence to Dr Franklin G Miller, Department of Bioethics, National Institutes of Health, 10 Center Drive, Bldg. 10, rm 1C118, Bethesda, MD 20892-1156, USA; FMiller@cc.nih.gov}

Received 21 August 2012 Revised 1 November 2012 Accepted 19 November 2012 Published Online First 13 December 2012

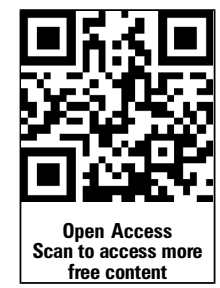

To cite: Brim RL, Miller FG. J Med Ethics 2013;39. 703-707.

\begin{abstract}
There has been considerable debate surrounding the ethics of sham-controlled trials of procedures and interventions. Critics argue that these trials are unethical because participants assigned to the control group have no prospect of benefit from the trial, yet they are exposed to all the risks of the sham intervention. However, the placebo effect associated with sham procedures can often be substantial and has been well documented in the scientific literature. We argue that, in light of the scientific evidence supporting the benefits of sham interventions for pain and Parkinson's disease that stem from the placebo effect, these sham-controlled trials should be considered as offering potential direct benefit to participants. If scientific evidence demonstrates the positive effect of placebo from sham interventions on other conditions, sham-controlled trials of interventions for the treatment of these conditions should be considered to have prospects of benefit as well. This potential benefit should be taken into account by research ethics committees in risk-benefit analyses, and be included in informed consent documents.
\end{abstract}

\section{INTRODUCTION}

Clinical trials evaluating new therapies often use placebo control groups as a way to measure treatment-specific efficacy. In drug trials, this generally takes the form of an inert pill that resembles the trial drug. However, in trials of new surgical techniques, medical procedures, implantations or manipulations, like acupuncture, a 'sham' intervention is often used as the control. The sham procedure mimics the actual procedure in every way, including preprocedure routine, anaesthesia and incisions (if necessary), and postprocedure follow-up. However, participants in the sham arm do not receive the procedure under investigation. Sham procedures have generally been considered a more appropriate control than using no physical intervention, or usual medical care, in trials evaluating the efficacy of new procedures. Sham procedures more clearly distinguish whether a new procedure is effective beyond the placebo response that is due to the contextual cues of an invasive procedure.

The ethics of using sham controls in trials of new procedures has been extensively discussed and debated in the literature with particular emphasis on new invasive therapies for Parkinson's disease (PD) and pain. ${ }^{1-4}$ A prevailing assumption among commentators both in favour of, and opposed to, sham-controlled trials is that participants receiving the sham intervention will have no prospect of benefit while being exposed to its risks. This is reflected in both the risk-benefit assessments made by research ethics committees (RECs) and the informed consent process.

However, the substantial scientific evidence supporting the therapeutic benefit of the placebo effect warrants rethinking our current practices relating to risk-benefit assessments and informed consent for sham-controlled trials of invasive procedures. Placebos can elicit strong physiological effects and produce meaningful symptomatic relief. Indeed, there is often more sound evidence on the benefits of placebos than there is for experimental treatments under investigation. The physiological basis of the placebo effect is well understood for certain conditions, and sham procedures generally produce greater placebo responses in clinical trials than pharmacological placebos. ${ }^{5}$ For the purposes of this article, we define the effects attributable to a placebo intervention as the 'placebo effect,' and the response of a participant in a control arm of a randomised trial as a 'placebo response.'

Despite growing scientific knowledge concerning placebo effects, investigators and RECs have continued to characterise sham procedures as 'nontherapeutic' interventions that carry risks to subjects without providing any prospect of benefit. There has been little consideration of whether the potential benefits from the placebo effect should be included in risk-benefit assessments, and whether the informed consent process should describe the positive aspects of the placebo effect to participants. A recent study of UK participant information leaflets found that only five of 45 leaflets mentioned that a placebo was capable of producing clinical effects. ${ }^{6}$

Current practices may reflect the negative connotation of 'placebo' in the medical literature. Placebo is often described as something inert, lacking specific mechanistic activity, and a scientific tool meant only to control for the contextual cues of a study and biases of researchers and participants. ${ }^{6}{ }^{7}$ By contrast, experimental therapies are referred to as 'active' treatments, genuine, real and 'the focus of the study'. ${ }^{7}$ Thus, it is evident that most researchers believe the only real potential benefit a participant may receive in a sham-controlled trial is from the investigational procedure.

The accumulation of scientific evidence on the placebo effect means it is time to reassess how the placebo effect is regarded in risk and benefit assessments of sham-controlled trials and during the 
informed consent process. Herein, we briefly discuss the current scientific data on the placebo effect, in general, and how this applies to sham-controlled trials of new therapies for PD and pain. We argue that the potential benefit from the placebo effect should be considered in risk-benefit assessments, and the informed consent process for sham-controlled trials of procedures to treat PD and pain. In addition to promoting accuracy in risk-benefit assessment and informed consent, rethinking the potential benefits from sham procedures resulting from placebo effects makes it easier to justify the evaluation of invasive procedures by means of sham-controlled trials.

\section{SCIENTIFIC EVIDENCE OF PLACEBO EFFECTS: IMPLICATIONS FOR INVASIVE PLACEBO CONTROLS}

The placebo effect is a physiological phenomenon, which varies with the invasiveness of the intervention, the nature of associated environmental cues, condition being treated and participants' expectation of receiving benefit. $^{8} 9$ The neurological basis of the placebo effect has been extensively studied, and we highlight here, the processes most relevant for PD and pain (more detailed and complete analysis can be found in references $\left.{ }^{7}{ }^{10-14}\right)$. The effect of placebo on pain is mediated, in part, by the release of endogenous opioids and endocannabinoids. ${ }^{10-12}$ Expectations also play a large role in the placebo effect; expectation of pain relief upon administration of a placebo is mediated by regions of the prefrontal cortex that can trigger endogenous opioid release. ${ }^{10}{ }^{11}$ Evidence also indicates that placebo interventions can divert attention away from pain. ${ }^{10}$ Additionally, expectation of relief can decrease pain via dopamine release in reward centres of the brain. ${ }^{13}$

Given the role of dopamine in the placebo effect, PD symptoms can be susceptible to the effects of placebo, since dopamine is diminished in PD. The magnitude of placebo-mediated dopamine release can be altered by manipulating participants' expectation of potential benefit, and can be equivalent to the dopamine release elicited by clinically used PD drugs. ${ }^{8} 14$ Participants in both arms of two separate sham-controlled surgical intervention trials aimed at reducing PD symptoms showed significant improvement, even though there was no difference in overall outcomes between the sham and intervention in either trial. $^{9} 15$ Patients who believed they received a real procedure had better symptom control by blind evaluation and selfreport. ${ }^{9}$ This trend was also observed in multiple trials of acupuncture versus superficial needling at non-acupuncture points (sham acupuncture) for pain relief; no difference was found between the arms, but the participants' expectations of pain relief predicted their response to treatment. ${ }^{16}$ Overall, the evidence suggests that PD symptom and pain relief can be strongly influenced by the placebo effect, and expectations are a good predictor of outcomes from placebo treatments.

To assess the overall clinical significance of placebo effects on responses to treatment, a large meta-analysis was conducted that included trials with investigational, placebo and no-treatment groups. ${ }^{5}$ As compared with no-treatment groups, placebo interventions did not have clinical significance; however, subgroup analyses revealed that placebo was effective in altering subjective patient-reported outcomes, especially for pain. Most importantly, this meta-analysis revealed that physical placebo interventions, such as sham acupuncture, were associated with larger responses than pharmacological placebos. ${ }^{5}$ Physical interventions can range from acupuncture to brain surgery, and the more invasive and stressful an intervention is, the greater the placebo response. $^{5}{ }^{17}$ However, it is important to acknowledge that the magnitude of the placebo effect from any particular sham procedure is unclear.

Sham-controlled trials of internal mammary artery ligation ${ }^{18}$ (to reduce angina pain), vertebroplasty ${ }^{19}$ (the injection of cement into fractured vertebra to reduce pain), and arthroscopic debridement of the knee ${ }^{20}$ (to reduce osteoarthritis pain), all demonstrated that sham surgery had substantial effects on patient-reported outcomes. Even though these procedures were being widely used in medical practice at the time of the trials, none of them resulted in better patient outcomes than sham surgery. In these particular cases natural history may have some explanatory power, as no-treatment groups were not included in these trials; however, the extensive data on pain reduction from the placebo effect, and the meta-analytic data demonstrating the enhanced placebo response created by physical sham procedures, make it reasonable to assume that sham-controlled trials offer a prospect of benefit.

Clinical trial results suggest that the duration of the placebo effect can be substantial. Pain relief from sham arthroscopy of the knee lasted through the 2-year postprocedure follow-up. ${ }^{20}$ Placebo-associated benefits in sham-controlled trials of new PD treatments have lasted several months. ${ }^{15}$ In a trial including 1162 patients with chronic low back pain randomised to traditional acupuncture, a sham-acupuncture and usual care, patients receiving the sham intervention had greater pain relief for at least 6 months as compared with those receiving usual medical care. ${ }^{21}$

\section{IMPLICATIONS OF CONSIDERING THE PLACEBO EFFECT IN RISK-BENEFIT ASSESSMENTS}

The prospect of placebo benefit should be considered by RECs in risk-benefit analyses for sham-controlled studies of procedures for medical conditions that have sufficient medical evidence supporting the placebo effect. In particular, for those conditions, like PD and pain with substantial evidence, the placebo effect produced by sham interventions should be considered as a potential benefit.

It may only be appropriate to consider the placebo effect as a benefit when the study outcome, or the method by which clinical improvement in the specific disease is measured, is subjective or modifiable by psychological factors, such as expectation (eg, exercise tolerance). The outcomes of PD symptom and pain relief are subjective. In other conditions, such as asthma, there are both subjective outcomes and quantifiable clinical endpoints, and trials have shown placebos improving patient-reported outcomes but not measurable clinical endpoints. ${ }^{22}$ It is sometimes assumed that subjective outcomes do not have the same weight as objective clinical endpoints, however, for conditions like PD and chronic pain, there are currently no disease-modifying treatments, and patients can only hope to modify their quality of life.

A main criticism of sham-controlled trials is that participants assigned to the sham intervention have no prospect of benefit while being exposed to all the risks of a procedure. Clinical trial design is supposed to minimise harm to participants and have a reasonable balance of risks and benefits. If the sham arm offers no prospect of benefit, and exposes subjects to substantial risks, the trial would have an unfavourable risk-benefit profile. This has led some bioethicists to reject sham-controlled trials:

It is undeniable that performing surgery in research subjects that has no potential therapeutic benefit fails to minimize the risks of harm. An alternative research design that did not involve sham surgery would pose a lower risk of harm to the subject in the control group. ${ }^{4}$

Using a sham surgery component ... adds risks of foreseeable and preventable harm without a corresponding benefit to subjects in the control arm. ${ }^{3}$ 
We contend that the scientific evidence supporting the placebo effect challenges the assumption that subjects randomised to sham-control interventions receive no potential benefit.

Resistance to considering sham procedures as benefits may be based on the intention of performing them. The intention of administering a sham procedure is to provide an indistinguishable comparison intervention for the purpose of rigorous evaluation of treatment efficacy, not to benefit participants. However, it might also be questioned whether researchers intend to benefit participants by administering an investigational treatment, since the purpose of randomised trials is to conduct scientific experiments aimed at informing clinical practice. Currently, treatments evaluated for efficacy by randomised trials (including both experimental therapies and non-validated procedures used in clinical practice) and standard-of-care control interventions are thought to offer a prospect of therapeutic benefit. By contrast, sham interventions are not being evaluated for efficacy. However, it does not follow that sham procedures lack the potential for therapeutic benefit. What grounds a prospect of benefit from a procedure is not the intent of administration, but the evidence in support of a causal connection between administering the procedure and positive outcomes for patients.

Investigational treatments are routinely considered to offer a prospect of benefit, often based on little solid evidence. This evidence is often data generated through animal models, which are often poor mimics of human disease states, and uncontrolled clinical trials that are often unreliable indicators of clinical benefit. For example, many investigational brain-delivered PD treatments, including neurotrophic factor, retinal-pigmented epithelial cells and neurturin, have shown potential benefit in preclinical testing and small uncontrolled safety and tolerability trials, but failed to show any benefit in larger sham-controlled studies. ${ }^{23}$ The evidence for potential benefit from these experimental treatments is, therefore, less rigorous than the controlled, clinical investigations conducted with several different techniques that have demonstrated sham procedures relieving pain and PD symptoms. Consequently, if receipt of an investigational treatment is considered a potential benefit, the placebo effect from a sham intervention should be considered a potential benefit as well.

Including all types of benefits, regardless of the source, is consistent with the US federal regulations governing research with human subjects (CFR 46.111(a)(2)). These regulations require that institutional review boards (IRBs) assure 'risks to subjects are reasonable in relation to anticipated benefits'. The regulations do not stipulate that only specific kinds of benefits should be considered.

The placebo effect should be regarded by RECs and IRBs as a potential benefit to patients receiving sham invasive interventions in trials of treatments for conditions that have sound medical evidence demonstrating placebo benefit to patients. Currently, pain and PD fall into this category, and the list will likely grow. Finally, considering potential benefit from the placebo effect would ease ethical concerns about sham-controlled trials insofar as there is evidence to support a prospect of benefit from sham procedures, which can at least partially offset the risks of these procedures.

\section{IMPLICATIONS OF CONSIDERING THE PLACEBO EFFECT IN INFORMED CONSENT}

A basic element of informed consent is a description of the potential risks and benefits from research procedures; hence, mentioning potential benefits from sham-controlled trials of treatments for PD and pain due to the placebo effect is required. The current approach to informed consent for participants entering randomised sham-controlled trials is to educate participants that their assignment to the sham arm will provide no benefit. This approach was used in the study of arthroscopic knee surgery for the treatment of osteoarthritis pain:

All patients provided informed consent, which included writing in their chart, 'On entering this study, I realize that I may receive only placebo surgery. I further realize that this means that I will not have surgery on my knee joint. This placebo surgery will not benefit my knee arthritis. ${ }^{20}$

The statement in this informed consent document was directly contradicted by the results of the study itself. All participants in the study had equivalent improvement regardless of their trial arm allocation, suggesting that the benefit patients received from the procedure was largely due to the placebo effect. Those receiving sham surgery, directly benefited from the trial with less adverse events reported compared with the procedure arm.

The process of informed consent should introduce participants to the placebo effect and explain how it may benefit them. This approach would more closely follow the US federal regulations (CFR 46.116 (a)), which state that informed consent should include '... a description of any benefits to the subject or to others which may reasonably be expected from the research ...' It has been suggested by others that informed consent disclosures should tell participants that the placebo effect is 'a real physiological phenomenon resulting from feelings, appropriate, beliefs, expectations, settings and interactions'. ${ }^{24}$ Disclosing information about the placebo effect in informed consent is consistent with the principle of respect for persons, as disclosure would provide greater exchange of information and allow participants to make more educated choices about enrolment in research.

Informing participants of the potential benefit from a sham intervention could have a variety of implications. First, participant recruitment may improve for sham-controlled trials. In the knee surgery trial cited above, only 180 (56\%) of the 324 eligible patients enrolled in the study. ${ }^{20}$ Participants' fear of the risks from an invasive intervention, which is described in detail, without any understanding of the potential benefit of the sham intervention likely, contributes to low participation in these kinds of trials. Reshaping language about the sham arm to more accurately reflect the potential benefit may help to enrol more patients in the research enterprise.

Second, informing patients about the placebo effect should not reduce it. ${ }^{4}$ This was demonstrated in an irritable bowel syndrome (IBS) trial, which informed participants that they would receive 'placebo pills made of an inert substance, like sugar pills, which have been shown in clinical studies to produce significant improvement in IBS symptoms through mind-body self-healing processes'. ${ }^{25}$ Individuals treated with placebo had higher symptom-improvement scores and decreased symptom severity than those who did not receive an intervention but had the same level of provider contact. In another study, healthy volunteers who experienced a placebo effect, and were informed of their reaction, were just as likely to receive placebo benefit again, compared with those who were not told. ${ }^{26}$ Both these cases support the notion that informing participants about the placebo effect does not undermine, and may increase, the potential benefit from the placebo effect; however, more research across a variety of conditions needs to be performed.

Lastly, informing participants about the potential benefits of placebo may have unintended consequences for study results. The placebo response in clinical trials can be enhanced by manipulating participants' expectations. For instance, a placebocontrolled study examining an asthma treatment presented participants with either basic materials about the benefits and risks 
of the therapy, or gave them additional positive messages about the value of the therapy. Subjects who received placebo treatment and positive 'enhanced messages' had greater improvements in patient-reported outcomes than subjects who did not receive 'enhanced messages'. ${ }^{22}$ By contrast, there was no effect of 'enhanced messages' on subjects who received the experimental treatment. This implies that the placebo response was not equally strong between the two arms of the trial, and cannot simply be subtracted out. Informing participants of the benefits of placebo before interventional studies may act like 'enhanced messages' which increase the placebo response in the sham arm, and thus, decrease the differences between arms, making the effect of the experimental procedure harder to tease out. ${ }^{24}$ Further studies are needed to establish whether these observations are generalisable to procedural intervention trials and to other disease states.

It is important that investigators take the possibility of altered expectation and enhanced placebo responses into consideration so that they can mitigate this risk and choose appropriate informed consent language. Informing participants about the placebo effect should use tempered language that is relatively neutral in order to avoid bias and unreasonably altering expectations. However, it should also accurately describe the placebo effect and the potential benefits from it. We suggest language similar to the following:

The trial you are enrolling in seeks to compare treatment $T$ for patients with condition $C$ to a control treatment called a 'sham', in which no $T$ will be performed. The sham is a comparison for $T$ and is not designed to benefit you; however, there is the possibility you may benefit from being assigned to either the treatment or the control 'sham' arm of the trial due to a real biological reaction your body has to being in a medical environment, and your own expectations of benefit from being enrolled in this trial. This reaction, called 'the placebo effect' has been shown to relieve some symptoms of $C$. The probability and magnitude of any benefit from either Tor sham is uncertain.

\section{CONCLUSIONS}

We have argued that the potential benefit to research participants receiving sham invasive procedures for PD or pain should be reflected in risk-benefit assessments conducted by RECs, and in informed consent documents and conversations. The evidence of positive placebo effects from sham interventions support attributing prospect of benefit to participants receiving these interventions within clinical trials. Although the magnitude of benefit a participant may receive from the sham procedure is not always clear, neither is the magnitude of benefit from the intervention under investigation.

Considering the placebo effect as a benefit to study participants in sham-controlled trials does not presume that all trials will have favourable risks-benefit profiles. When considering the ethics of an individual sham-controlled trial, the risks and benefits specific to that trial need to be weighed. There are trials that will not meet favourability criteria, even after consideration of the potential benefit from the placebo effect; however, when relevant to the condition under study, the placebo effect should be included in the analysis to assure that it is rigorous and scientifically accurate.

Our argument to include the placebo effect as a benefit in clinical research does not necessarily apply to clinical practice. There is widespread debate, and a broad literature, about the use of placebos in medicine, although $86 \%$ of general practice physicians and $57 \%$ of internists and rheumatologists report using a placebo treatment in the past year. ${ }^{27}$ Clinical care and clinical research have different goals, standards and regulations. For instance, exposing subjects to treatments with unknown benefits and risks, and performing tests and procedures that are not done for the care of the patient, are acceptable in research but not clinical care, because the goal of research is to gain knowledge. We believe that the use of placebos in clinical trials is consistent with the goals, standards and regulations of research, and that the placebo effect qualifies as a potential benefit to patients in sham-controlled trials.

More research on consent language, and the views of RECs about the placebo effect, will help determine the best course of action to begin using scientific evidence to better inform decisions about which interventions have a prospect of benefit in sham-controlled trials.

Acknowledgements Thanks to Drs Steven Pearson, David Wendler and Tina Rulli for their insightful commentary and assistance during the editing process.

Contributors RLB and FGM developed the original idea for the article. RLB wrote the initial manuscript, and both authors contributed to revisions of drafts of the manuscript, including the final version.

Funding This research was supported by the intramural research program of the National Institutes of Health Clinical Center.

\section{Competing interests None.}

Provenance and peer review Not commissioned; externally peer reviewed.

Open Access This is an Open Access article distributed in accordance with the Creative Commons Attribution Non Commercial (CC BY-NC 3.0) license, which permits others to distribute, remix, adapt, build upon this work non-commercially, and license their derivative works on different terms, provided the original work is properly cited and the use is non-commercial. See: http://creativecommons.org/ licenses/by-nc/3.0/

\section{REFERENCES}

1 Miller FG, Kaptchuk TJ. Sham procedures and the ethics of clinical trials. J R Soc Med 2004;97:576-8.

2 Miller FG, Wendler $\mathrm{D}$. The ethics of sham invasive intervention trials. Clin Trials 2009:6:401-2.

3 London AJ, Kadane JB. Placebos that harm: sham surgery controls in clinical trials. Stat Methods Med Res 2002;11:413-27.

4 Macklin R. The ethical problems with sham surgery in clinical research. N Engl J Med 1999;341:992-96.

5 Hróbjartsson A, Gøtzsche P. Placebo interventions for all clinical conditions. Cochrane Database of Systematic Reviews 2010;1:CD003974.

6 Bishop FL, Adams AE, Kaptchuk TJ, et al. Informed consent and placebo effects: a content analysis of information leaflets to identify what clinical trial participants are told about placebos. PLoS One 2012;7:e39661.

7 Miller FG, Brody $\mathrm{H}$. Understanding and harnessing placebo effects: clearing away the underbrush. Journal Med Philos 2011;36:69-78.

8 Lidstone SC, Schulzer M, Dinelle K, et al. Effects of expectation on placebo-induced dopamine release in Parkinson disease. Arch Gen Psychiatry 2010;67:857-65.

9 McRae C, Cherin E, Yamazaki TG, et al. Effects of perceived treatment on quality of life and medical outcomes in a double-blind placebo surgery trial. Arch Gen Psychiatry 2004:61:412-20.

10 Wager TD, Rilling JK, Smith EE, et al. Placebo-induced changes in FMRI in the anticipation and experience of pain. Science 2004;303:1162-7.

11 Amanzio M, Benedetti F. Neuropharmacological dissection of placebo analgesia: expectation-activated opioid systems versus conditioning-activated specific subsystems. J Neurosci 1999;19:484-94.

12 Benedetti F, Amanzio M, Rosato R, et al. Nonopioid placebo analgesia is mediated by CB1 cannabinoid receptors. Nat Med 2011;17:1228-30.

13 Scott DJ, Stohler CS, Egnatuk CM, et al. Individual differences in reward responding explain placebo-induced expectations and effects. Neuron 2007;55(2):325-36.

14 de la Fuente-Fernandez R, Ruth TJ, Sossi V, et al. Expectation and dopamine release: mechanism of the placebo effect in Parkinson's disease. Science 2001;293:1164-6.

15 Goetz CG, Leurgans S, Raman R. Placebo-associated improvements in motor function: comparison of subjective and objective sections of the UPDRS in early Parkinson's disease. Mov Disord 2002;17:283-8.

16 Linde K, Witt CM, Streng A, et al. The impact of patient expectations on outcomes in four randomized controlled trials of acupuncture in patients with chronic pain. Pain 2007:128:264-71.

17 Beecher HK. Surgery as placebo. A quantitative study of bias. JAMA 1961;176:1102-7. 
18 Dimond EG, Kittle CF, Crockett JE. Comparison of internal mammary artery ligation and sham operation for angina pectoris. Am J Cardiol 1960;5:483-6.

19 Kallmes DF, Comstock BA, Heagerty PJ, et al. A randomized trial of vertebroplasty for osteoporotic spinal fractures. N Engl J Med 2009;361:569-79.

20 Moseley JB, O'Malley K, Petersen NJ, et al. A controlled trial of arthroscopic surgery for osteoarthritis of the knee. $N$ Engl J Med 2002;347:81-8.

21 Haake M, Muller HH, Schade-Brittinger C, et al. German Acupuncture Trials (GERAC) for chronic low back pain: randomized, multicenter, blinded, parallel-group trial with 3 groups. Arch Intern Med 2007;167:1892-8.

22 Wise RA, Bartlett SJ, Brown ED, et al. Randomized trial of the effect of drug presentation on asthma outcomes: the American Lung Association Asthma Clinical Research Centers. J Allergy Clin Immunol 2009;124:436-44.
23 Katsnelson A. Experimental therapies for Parkinson's disease: why fake it? Nature 2011;476(7359):142-4.

24 Bishop FL, Jacobson EE, Shaw J, et al. Participants' experiences of being debriefed to placebo allocation in a clinical trial. Qual Health Res 2012;22:1138-49.

25 Kaptchuk TJ, Friedlander E, Kelley JM, et al. Placebos without deception: a randomized controlled trial in irritable bowel syndrome. PLoS One 2010;5(12): e15591.

26 Chung SK, Price DD, Verne GN, et al. Revelation of a personal placebo response: its effects on mood, attitudes and future placebo responding. Pain 2007;132:281-8

27 Miller FG, Colloca L. The legitimacy of placebo treatments in clinical practice: evidence and ethics. Am J Bioeth 2009:9:39-47. 